Massimo Bellomi

Patrick Maisonneuve

Sara Raimondi

Giulia Veronesi

\section{Reply to Letter to the Editor re: Evolution of emphysema in relation to smoking}

Received: 14 December 2009

Accepted: 16 December 2009

Published online: 24 February 2010

(C) European Society of Radiology 2010

This reply refers to the Letter to the Editor available at doi:10.1007/s00330010-1728-4

\footnotetext{
M. Bellomi $(\bowtie)$

European Institute of Oncology, Department of Radiology,

435, Via Ripamonti,

20141 Milan, Italy

e-mail: massimo.bellomi@ieo.it

Tel.: +39-2-57489041

Fax: +39-2-57489040

P. Maisonneuve $\cdot$ S. Raimondi Department of Epidemiology and Biostatistics,

European Institute of Oncology, Milan, Italy

G. Veronesi

Department of Thoracic Surgery, European Institute of Oncology, Milan, Italy
}

Dear Sir,

The letter "Optimizing the assessment of the longitudinal behaviour of emphysema via MDCT" draws attention to some issues regarding our cited article.

First, we agree that differences in the level of inspiration between baseline and repeat MDCT could affect density measurements. This is the reason why we adjusted our analysis for difference in MDCT lung volume measurements between the two procedures. However, we do not believe that differences in the level of inspiration represent a real confounding factor for our main findings since lung volume variation was not associated with smoking status $(p=0.81$ using absolute values and $p=0.96$ using log-transformed values).

Finally, we want to clarify why we did not use the absolute values of emphysema score, but only the percentage changes. The subjects enrolled in our study were selected among consecutive participants of a large-scale lung cancer screening trial. The subjects' age at baseline MDCT varied from 50 to 74 years; some were completely asymptomatic while others had a history of pulmonary disease or dyspnoea. As a result, the absolute value of emphysema score varied widely among participants, with values ranging from 0.2 to 25 (using a threshold of $-930 \mathrm{HU})$. In addition we showed that the absolute values of the emphysema score measured at baseline MDCT varied significantly according to the smoking status, age, gender and occupational exposure of the participants. Therefore, we only assessed the percentage change of emphysema score in order to adjust for baseline emphysema score. 